\title{
An Adaptive Tone Mapping Algorithm for High Dynamic Range Images
}

\author{
Jian Zhang and Sei-ichro Kamata \\ Graduate School of Information, Production and Systems, Waseda University \\ 2-7 Hibikino, wakamatsu-ku, kitakyushus-shi, Fukuoka 808-0135, Japan \\ zj_jay@toki.waseda.jp, kam@waseda.jp \\ http://www.waseda.jp/ips
}

\begin{abstract}
Real world scenes contain a large range of light intensities which range from dim starlight to bright sunlight. A common task of tone mapping algorithms is to reproduce high dynamic range(HDR) images on low dynamic range(LDR) display devices such as printers and monitors. In this paper, a new tone mapping algorithm is proposed for the display of HDR images. Inspired by the adaptive process of the human visual system, the proposed algorithm utilized the center-surround Retinex processing. The novelty of our method is that the local details are enhanced according to a non-linear adaptive spatial filter (Gaussian filter), whose shape is adapted to high-contrast edges of the image. The proposed method uses an adaptive surround instead of the traditional pre-defined circular. Therefore, the algorithm can preserve visibility and contrast impression of high dynamic range scenes in the common display devices. The proposed method is tested on a variety of HDR images, and we also compare it to previous work. The results show good performance of our method in terms of visual quality.
\end{abstract}

Keywords: Tone mapping, retinex, high dynamic range, Hilbert scan, Gaussian filter.

\section{Introduction}

HDR imaging techniques can produce images that record the full dynamic range of the scene in the real world. Recently, HDR images can be captured easily using a composition of multiple photographs of the same scene with different exposures [1] or new sensor technologies [2]. Thus, the availability of HDR data becomes more commonplace. In contrast, HDR display devices are not commonplace yet because of their high price. Furthermore, most current output devices, such as computer monitors and printers, often only make a part of the scene visible, which is much lower than the dynamic range of the scene. This mismatch between HDR input and LDR output leads to the problem that how we can reproduce or render such images in a standard output device. In order to solve this problem, tone mapping techniques[3]-[6] are used to map HDR values to $\mathrm{LDR}$ values. Tone mapping is a contrast enhancement technique which can

A. Trémeau, R. Schettini, and S. Tominaga (Eds.): CCIW 2009, LNCS 5646, pp. 207-215, 2009.
(C) Springer-Verlag Berlin Heidelberg 2009 


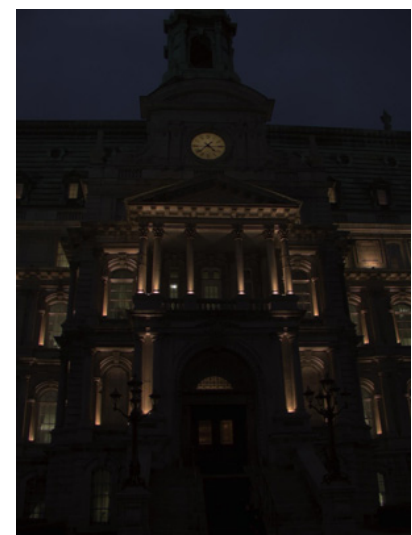

(a) Linear scaling



(b) Image treated with our method

Fig. 1. An example of HDR image that needs a tone mapping technique

scale the HDR data in order to preserve certain characteristics of the input HDR image, such as contrast, visibility or appearance in the resulting displayable image. Currently, there existed a number of tone mapping algorithms. According to the transformation they apply to convert input luminance to the output values, they are usually categorized into two main groups, i.e., global operators and local operators.

Global tone mapping methods compress the original range into the output range in accordance with non-linear one-to-one matching functions, for example, logarithmic function, gamma function, sigmoidal function and so on [3]. These global algorithms tend to preserve the subjective perception of the scene and have advantage of being simple and fast. However, simple global processing will cause a loss of contrast, which is apparent in the loss of detail visibility in the dark or light parts of the image (e.g. Fig.1). So they are still difficult to reproduce all the visual details. On the other hand, local tone mapping methods refers to the algorithms which use a local processing for reproduction. These methods are not based on a one-to-one mapping. The mapping function is changed according to the spatial context of the scene, therefore two different pixels with the same intensity in the original image can be mapped to different display values. This makes the local operator-based methods give more details of the image than those global operator-based methods. However, they may also cause "halo" effects or ringing artifacts in the reproduction.

The Human Visual System (HVS) can perceive a vast range of intensities well by using various adaptation mechanisms. Land's Retinex is a simplified model of the HVS and can be adapted easily for computational image rendering algorithms. Now, more evidence shows Retinex is an optimal solution to the lightness problem. In this paper, an adaptive tone reproduction algorithm based on the center-surround Retinex model is proposed. In general, our method belongs to the local operator's category. Compared to the previous surround-based 
algorithms, our Retinex-based local processing uses an adaptive surround instead of the traditional pre-defined circular. Because the shape of a surround can be changed according to the high-contrast edges, the proposed method does not generate halo artifacts but does preserve visibility of local details. Therefore, the proposed method can render high dynamic range images that models the adaptation of HVS. It has been implemented and tested in an image database. The experimental results show that this algorithm is effective and easy to use, only one parameter needs setting.

\section{The New Adaptive Processing Algorithm}

In this section, an adaptive tone mapping algorithm is presented. The proposed approach can overcome the problems mentioned above while achieving satisfactory results. Our work is totally focused on the luminance channel, so at first we introduce how to calculate the luminance values. Then the Retinex-based adaptive Gaussian filtering method is proposed. The local operators make use of a local information in the spatial domain, i.e., the shape of Gaussian filter should depend on the pixel's partial derivatives. This makes it reproduce the details of the image effectively. Note that all the operations are performed in logarithm space.

\subsection{Retinex-Based Local Processing}

Because we only treat the luminance in our method, we compute first the luminance $L$ (in $c d / m^{2}$ ) from the RGB space. In the LHS (luminance, hue, and saturation) system, the luminance is defined as

$$
L=0.299 R+0.587 G+0.114 B
$$

Let $L$ be the luminance image encoded linearly, whose maximum value is 1 .

Compared to the early stage of HVS where a global adaptation happens, the global tone mapping is performed for a first compression of the dynamic range. Then, we assume the logarithmic relation in our tone mapping solution, which can be described as the first luminance adjustment of HVS. The model presented in [7] essentially preserves good global perception. The following equation shows this mapping relationship.

$$
L^{\prime}=\frac{\log (L+\varphi)-\log \left(L_{\min }+\varphi\right)}{\log \left(L_{\max }\right)-\log \left(L_{\min }+\varphi\right)}
$$

In the above equation, $L^{\prime}$ is the nonlinear output luminance image, $L_{\max }$ and $L_{\text {min }}$ are the maximum and minimum luminance of the scene, and $\varphi$ is a small value to avoid the singularity that occurs if black pixels are present in the image and to control the overall brightness of the reproduced image. For example, when $\varphi$ decreases, the display will be bright and this leads to increase the sensitivity for dark areas. The parameter $\varphi$ is usually called "key value", because it decides 
whether an input value is mapped to a higher or lower value. In our research, we set it according to the average luminance $L_{a}$ which is calculated by

$$
\varphi=L_{a}=\exp \left(\frac{1}{N} \sum_{x=0}^{X} \sum_{y=0}^{Y} \log (L(x, y))\right)
$$

where $N$ is the number of pixels in the luminance image, an order tern $(x, y)$ of integers $x$ and $y$ is the coordinates of a pixel, $0 \leq x<X$ and $0 \leq y<Y$.

After a simple global processing, Retinex-based local processing is carried out. Traditionally, the Retinex is a member of the class of center surround functions where the output value of the function is obtained by computing the difference between the log-encoded treated pixel (center) and the log-encoded value of its neighborhood (surround). Here the surround is a Gaussian function. The mathematical form of the single-scale Retinex is defined as

$$
L_{\text {out }}(x, y)=\log \left(L^{\prime}(x, y)\right)-\log \left(G(x, y) * L^{\prime}(x, y)\right)
$$

where $L_{\text {out }}$ is the Retinex output image, $\log$ is the natural logarithm function. The symbol "*" is convolution operator. $G$ is a Gaussian filter (kernel) expressed as

$$
G(x, y)=\frac{1}{\sqrt{2 \pi} \sigma} \exp \left(-\left(x^{2}+y^{2}\right) / 2 \sigma^{2}\right)
$$

where $\sigma^{2}$ is the variance of Gaussian filter, and the size of the filter is often determined by $\sigma^{2}$. In the traditional Retinex [5], the value of sigma is fixed. However, it is known that an image with high-contrast edges are more pleasing to the human eyes, and that the human visual system is sensitive to the position of edges with high contrast in smooth areas. Thus if the fixed $\sigma^{2}$ is small, local contrast can be increased significantly while producing halo artifacts along highcontrast edges. On the contrary, a large $\sigma^{2}$ reduces the artifacts, but provides less increase in local contrast [8]. There exists a tradeoff between the increase in local contrast and good rendition of the image. So we make the filter variance adapt to the local characteristics of an image, that is to say, our Gaussian filter $G(x, y)$ is a function of the variable $\sigma(x, y)$. This is based on the strategy that different parts of an image should be smoothed differently, depending on the type of edges. The following equation shows information about contrast in horizontal, vertical and diagonal directions respectively.

$$
\begin{aligned}
& \nabla L^{\prime}(x, y)=L^{\prime}(x-1, y-1)+L^{\prime}(x+1, y-1)+L^{\prime}(x+1, y+1)+ \\
& L^{\prime}(x+1, y-1)-L^{\prime}(x-1, y)-L^{\prime}(x+1, y)-L^{\prime}(x, y-1)-L^{\prime}(x, y+1) .
\end{aligned}
$$

So in an area with high-contrast edges, the partial derivatives $\left(\nabla L^{\prime}\right)$ are large and the filter variance should be adapted to a small values to keep the high contrast along edges. On the other hand, in a smooth area the partial derivatives are small and the variance should be large to reduce artifacts. This illustrates a fact that there exists a proportional relationship between $\nabla L^{\prime}$ and $\sigma^{2}$. Therefore, we 
propose the following equation to calculate the variance of 2-D Gaussian filter at location $(x, y)$.

$$
\sigma^{2}(x, y)=\frac{\beta}{\left|\nabla L^{\prime}(x, y)\right|}
$$

where $\beta$ is a scaling factor, $|x|$ is absolute value of $x$ which insures $\sigma^{2}$ is a positive value.

We employ the 2 -D $(2 w+1) \times(2 w+1)$ adaptive Gaussian filter for $L^{\prime}(x, y)$, then obtain

$$
G(x, y) * L^{\prime}(x, y)=\frac{\sum_{x_{t}=x-w}^{x+w} \sum_{y_{t}=y-w}^{y+w} L^{\prime}\left(x_{t}, y_{t}\right) \cdot\left(\delta\left(x_{t}, y_{t}\right) \cdot \exp \left(\frac{-\left(x_{t}^{2}+y_{t}^{2}\right)}{\sigma^{2}(x, y)}\right)\right)}{\sum_{x_{t}=x-w}^{x+w} \sum_{y_{t}=y-w}^{y+w} \exp \left(\frac{-\left(x_{t}^{2}+y_{t}^{2}\right)}{\sigma^{2}(x, y)}\right)}
$$

Except adjusting variance $\sigma^{2}(x, y)$, the adaptive filter method should be able to adapt the shape of the filter to the high-contrast edges in the image in order to prevent halo artifacts. In other words, adaptive Gaussian filter doesn't blur the edges. So we use a weight number $\delta\left(x_{t}, y_{t}\right)$ to adapt the shape of the Gaussian filter in $(8) . \delta\left(x_{t}, y_{t}\right)$ changes the shape of the filter based on the information of high-contrast edges, and it can be calculated by

1) If $\left(x_{t}, y_{t}\right) \in \mathrm{I}, \quad \delta\left(x_{t}, y_{t}\right)=|| \nabla L^{\prime}\left(x_{t}, y_{t}\right)\left|-\delta\left(x_{t}-1, y_{t}-1\right)\right|+\left|\nabla L^{\prime}\left(x_{t}, y_{t}\right)\right|$,

$2)$ If $\left(x_{t}, y_{t}\right) \in \mathrm{II}, \quad \delta\left(x_{t}, y_{t}\right)=|| \nabla L^{\prime}\left(x_{t}, y_{t}\right)\left|-\delta\left(x_{t}, y_{t}-1\right)\right|+\left|\nabla L^{\prime}\left(x_{t}, y_{t}\right)\right|$,

8) If $\left(x_{t}, y_{t}\right) \in \mathrm{VIII}, \quad \delta\left(x_{t}, y_{t}\right)=|| \nabla L^{\prime}\left(x_{t}, y_{t}\right)\left|-\delta\left(x_{t}-1, y_{t}\right)\right|+\left|\nabla L^{\prime}\left(x_{t}, y_{t}\right)\right|$,

where I,II, $\cdots$,VIII describe eight regions of a $(2 w+1) \times(2 w+1)$ window, which is shown in Fig.2.

The output value of adaptive Gaussian filter centered at pixel $(x, y)$ is given by a weighted average of pixels surrounding the position $(x, y)$. And the weights are given by a 2-D adaptive Gaussian function, whose spatial constant and shape varies in accordance with the image high-contrast edges. From Equation (8), in

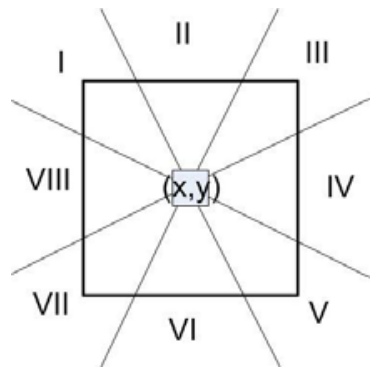

Fig. 2. Eight regions of of a $(2 w+1) \times(2 w+1)$ window 
fact it is done by adapting the variance $\sigma^{2}(x, y)$ and shape of the filter $\delta\left(x_{t}, y_{t}\right)$. According to Equation (6) we can get $\nabla L^{\prime}(x, y)$ for each pixel, then they are scaled to a larger range $\left[\min \left(\frac{\nabla L_{\min }^{\prime}}{\nabla L_{\max }^{\prime}}, 10^{-5}\right), 1\right]$, where $\nabla L_{\min }^{\prime}$ and $\nabla L_{\text {max }}^{\prime}$ mean the minimum and maximum $\nabla L^{\prime}(x, y)$ in a image, and function $\min ()$ is in order to perform division operator in (7).

In this study, we limit the size of the surround by taking parameter $\beta$ (in Equation (7)) into account. If we set a default value for the size $w$, then we can calculate the scaling factor $\beta$ as:

$$
\beta=\left(\nabla L_{\min }^{\prime} * w / 3\right)^{2}
$$

In our study, the default value of $w$ is 60 , then $\sigma(x, y)$ is scaled a range:

$$
\left[20 /\left(\min \left(\frac{\nabla L_{\min }^{\prime}}{\nabla L_{\max }^{\prime}}, 10^{-5}\right)\right), 20\right] .
$$

Figure 3 shows an example of the construction of our adaptive filter.

The reproduced luminance values are in the range $[0,1]$, but the output image still contains the very high dynamic range information even ignoring numerical errors. Thus, a quantization processing is required before the luminance is integrated back into the color image processing. This is done by histogram equalization technique (scaling and clipping). Then the original range is divided into $\mathrm{N}$ $(=255)$ intervals based on the pixel distribution. After the luminance processing applications, new luminance values are integrated back into the color image.



(a) An image with a Highcontrast edge

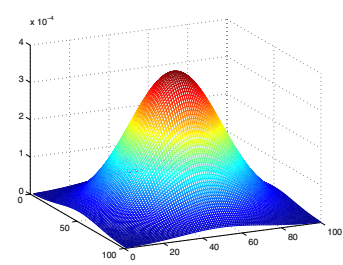

(c) General Gaussian filter

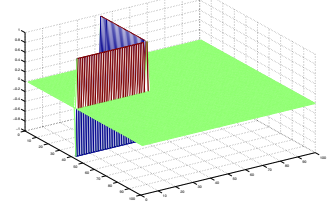

(b) $\nabla L^{\prime}$

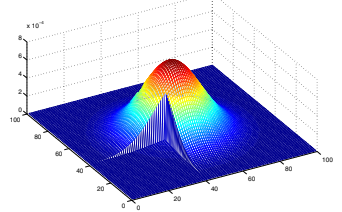

(d) The proposed adaptive Gaussian filter

Fig. 3. An example of the adaptive filter 


\section{Experiments}

In our simulation, we compute the HDR luminance image by Equation (1) firstly. Then the HDR luminance is mapped into the display luminance by the global compression function (2). Thirdly, based on the adaptive Gaussian filtering, each pixel is adjusted locally. Finally, the LDR image is rendered. Because it is very difficult to know how the light or dark the image should be displayed to be faithful to the original HDR image [3], until now, there are not any standard of objective evaluation available for measuring the quality of displayed HDR images. Main evaluation is based on human's subjective evaluation. However, the method which can increase the contrast while prevent halo artifacts may be desirable for certain applications. In this paper, we consider this to evaluate

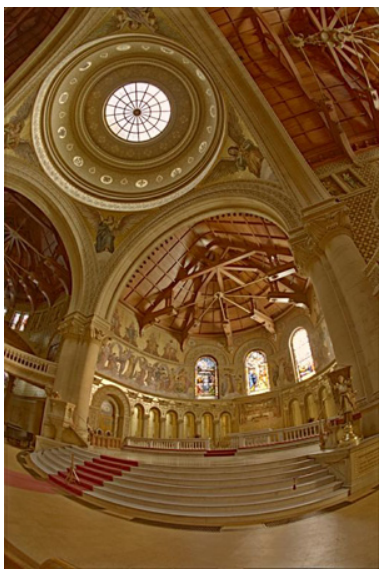

(a) The proposed method

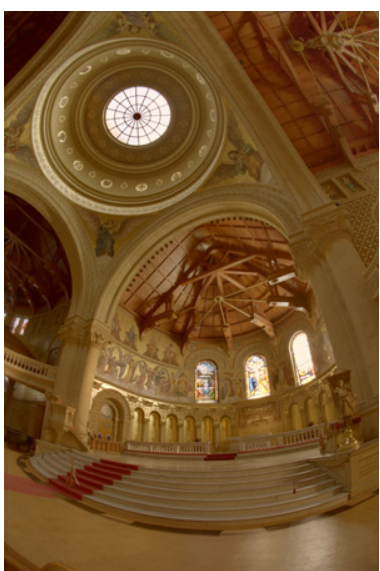

(c) Meylan's approach

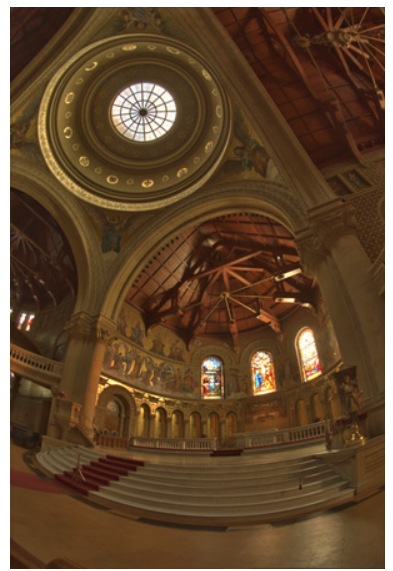

(b) Bilateral filtering

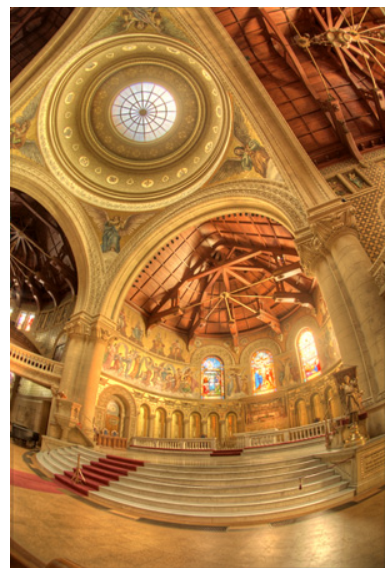

(d) Gradient comression

Fig. 4. The MemorialChurch image treated with different methods 


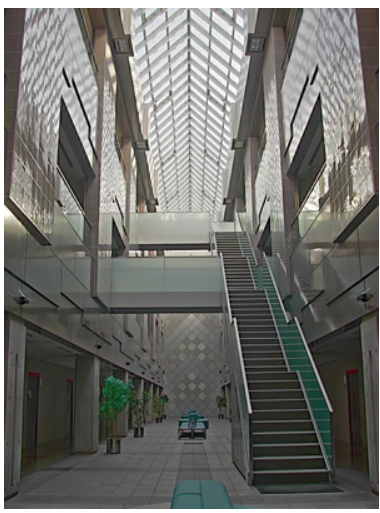

(a) The proposed method

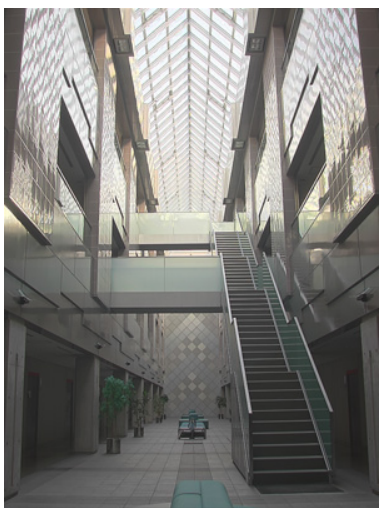

(c) Photographic mapping

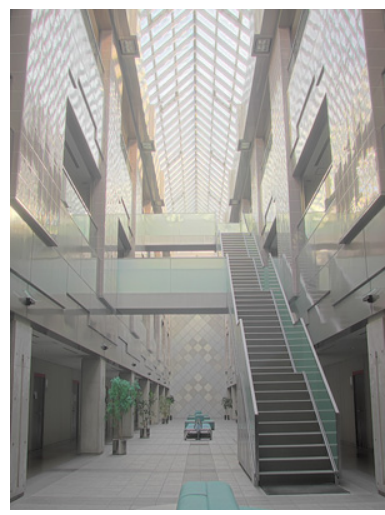

(b) Ashikhmin's approach

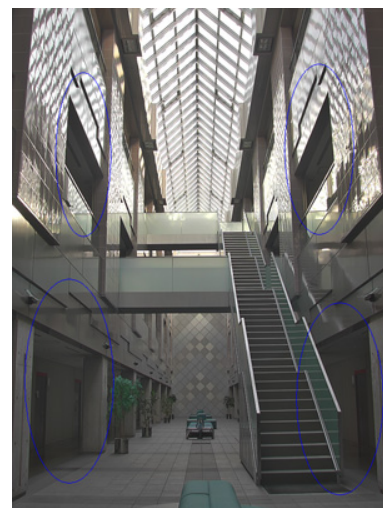

(d) Bilateral filtering

Fig. 5. The Atrium image treated with different methods

the performances of different algorithms. In Fig.4 and 5, we compare our algorithm with other local mapping methods. Fig.4(a) shows an image processed by our algorithm. When compared to bilateral filtering method and Meylan's method as shown in Figs.4(b) and (c), our algorithm can preserve more visual details in both the dim and bright areas under a good overall impression, especially on the left and right-up corners of the image. Fig.4(d) shows the result obtained by gradient domain contrast compression. This method is also good at increasing local contrast, however, the overall impression is different from the real-world scenes. Figs.5 (a) and (d) (regions in blue circles) show the difference between using an adaptive filter and a non-adaptive filter (the variance of Gaussian filter is a fixed value). We can find clearly that the image treated with the adaptive filter can preserve more visual details in both the dim and the bright regions, this is because adaptive processing can preserve high-contrast edges. 


\section{Conclusions}

A new adaptive tone mapping algorithm is proposed for rendering the HDR images in this paper. The proposed method provides an adaptively changed rule of the Gaussian filter's shape in tone mapping, this idea is based on the way by which the human eye perceives real-word scenes. The shape of a surround can be changed according to the high-contrast edges, which makes the proposed method not generate halo artifacts while increase the visibility of local details in the LDR images. Also, we tested our method on various HDR images, compared it with other approaches, and the results have demonstrated the effectiveness of the new method.

\section{References}

1. Debevec, P.E., Malik, J.: Recovering high dynamic range radiance maps from photograhs. In: SIGGRAPH 1997, pp. 369-378 (1997)

2. Seetzen, H., Whitehead, L.A., Ward, G.: A high dynamic range display using low and high resolution modulators. In: The Society for Information Display International Symposium (2003)

3. Reihhard, E., Stark, M., Shirley, P., Ferwerda, J.: Photographic Tone Reproduction for Digital Images. ACM Trans. on Graphics 21(3), 267-276 (2002)

4. Devlin, K.: A review of tone reproduction techniques. Computer Science, University of Bristol, Tech. Rep. CSTR-02-005 (2002)

5. Jobson, D.J., Rahman, Z., Woodell, G.A.: A multiscale retinex for bridging the gap between color images and the human obervation of scenes. IEEE Transaction on Image Processing 6(7), 965-976 (1997)

6. Tumblin, J., Rushmeier, H.: Tone reproduction for realistic image. IEEE Computer Graphics \& Applications 13(6), 42-48 (1993)

7. Qiu, G., Duan, J.: An optimal tone reproduction curve operator for the display of high dynamic range Images. In: IEEE ISCAS 2005, No. 6, pp. 6276-6279 (2005)

8. Meylan, L., Susstrunk, S.: High dynamic range image rendering with a Retinexbased adaptive filter. IEEE Transaction on Image Processing 15(9), 1820-1830 (2006) 